(Wiley, 2010).

6. Welch, G. C., San Juan, R. R., Masuda, J. D. \& Stephan, D. W. Science 314, 1124-1126 (2006).

7. Welch, G. C. \& Stephan, D. W. J. Am. Chem. Soc. 129, 1880-1881 (2007).
8. Chase, P. A., Welch, G. C., Jurca, T. \& Stephan, D. W. Angew. Chem. Int. Edn 46, 8050-8053 (2007).

9. Stephan, D. W. \& Erker, G. Angew. Chem. Int. Edn 54, 6400-6441 (2015)

10.Spielmann, J., Buch, F. \& Harder, S. Angew. Chem.
Int. Edn 47, 9434-9438 (2008).

11.Causero, A. et al. Organometallics 35, 3350-3360 (2016).

This article was published online on 8 January 2018.

\section{An ode to gene edits that prevent deafness}

\begin{abstract}
Gene editing can prevent inherited deafness in mice by disabling a mutant version of a gene that causes hearing loss. Is this a turning point on the path towards treating some types of human deafness? SEE LETTER P.217
\end{abstract}

\section{FYODOR URNOV}

$\mathrm{W}$ hen the 32-year-old composer Ludwig van Beethoven realized that his hearing was failing, he wrote to his brothers that "as the leaves of autumn wither and fall, so has my own life become barren". Although the cause of Beethoven's deafness is unknown, there are many examples of hearing loss in later life that are linked to inherited DNA changes. Two centuries later, techniques to prevent inherited forms of deafness are finally getting closer to implementation in the clinic. On page 217, Gao et al. ${ }^{1}$ report progress in using gene-editing technology to treat a mouse model of inherited deafness. Given the growing momentum in using genetic engineering for human therapy, the path needed to take this approach to the clinic is clear.

The remarkable process of sensing sound occurs in the inner ear ${ }^{2}$. Tiny, hair-like structures called cilia on the surface of hair cells in the cochlea respond to sound waves. Ciliary motion evokes an electrical signal because the properties of a protein assembly at the base of each cilium change when such motion occurs. The TMC1 protein is thought ${ }^{3}$ to be part of this assembly in humans, and some TMC1 mutations cause people to lose their hearing over time. The symptoms start in childhood, and deafness, along with associated degeneration and death of hair cells, ensues within 10 to 15 years ${ }^{4}$.

Gao and colleagues analysed the Beethoven mouse strain, in which the animals have a Tmc1 mutation that causes them to grow deaf over time ${ }^{5}$. The mouse mutation they studied matches a mutation in human TMC1 that is also linked to progressive hearing loss ${ }^{6}$. The mutation is dominant, which means that even if only one of a person's two copies of the gene has the mutation, they will become deaf. The mutant copy of the gene produces a defective protein that somehow impairs cell function, even though the cell also has a wild-type copy of the gene ${ }^{7}$

The repair of dominant-mutationassociated deafness is a delicate matter - the mutated gene must be disabled while preserving the wild-type gene within the same cell. This is no trivial undertaking, because only one nucleotide of DNA distinguishes the two versions of the TMC1 gene from each other (Fig. 1). One way to understand this is to imagine a duet between two people trying to sing in unison. If one person is off-key, this offender must be selectively silenced to allow the correct tune to be heard, because if both singers are stopped, the music will cease.

Gene editing is the technique of choice to rid a hair cell of the mutant version of a gene ${ }^{8}$. This involves using a nuclease enzyme to cut a targeted DNA sequence in the specific gene inside the living cell. The cut causes a doublestrand DNA break and the repair process often results in mistakes in which nucleotides are added or lost. Such a change can alter the sequence in a way that might cause translation to prematurely arrest and thereby prevent gene expression.

The authors used the nuclease Cas9, which cuts DNA at a specific site by using a snippet

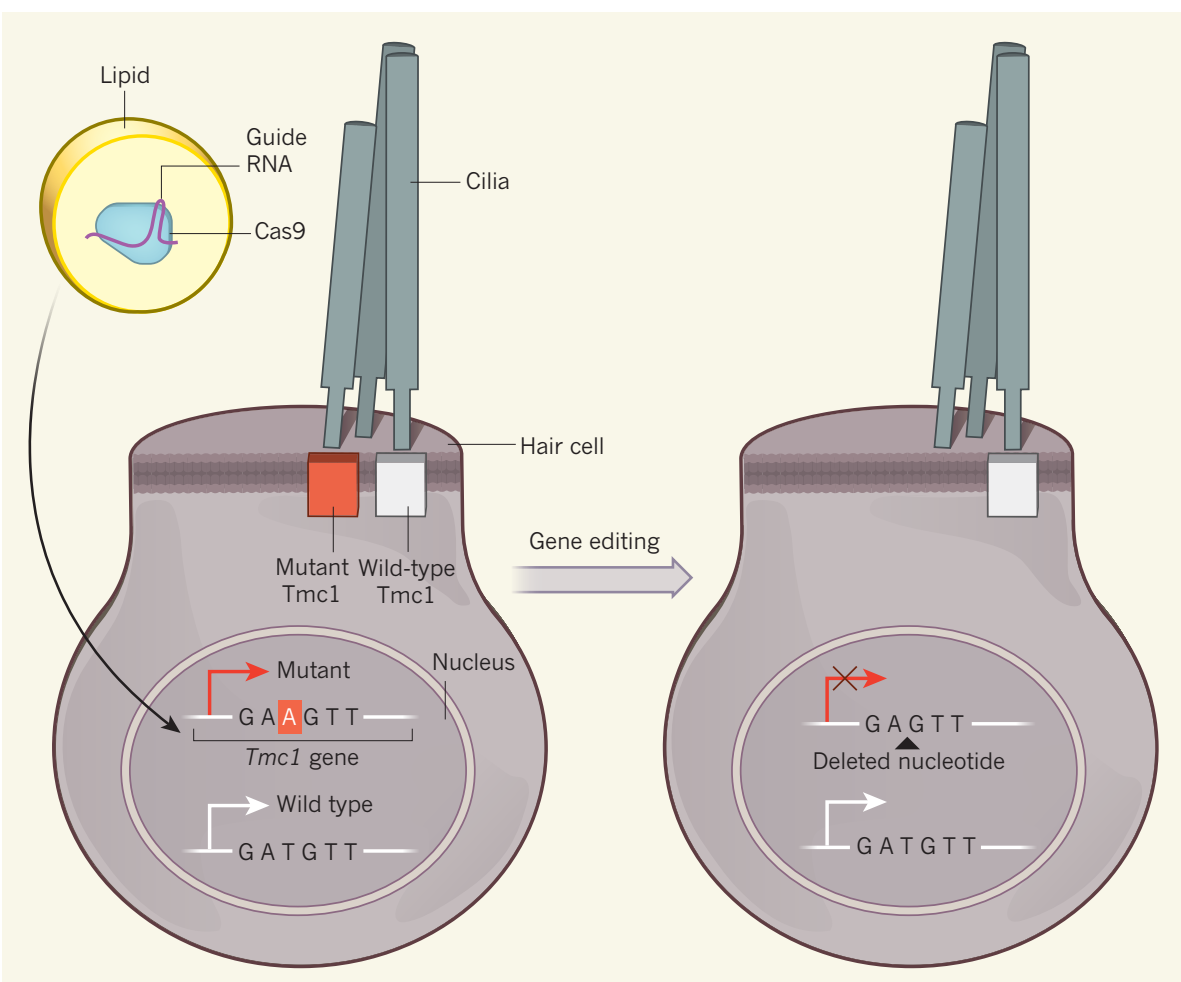

Figure 1 | Gene editing in mice can prevent inherited hearing loss. Gao $\mathrm{et}$ al. ${ }^{1}$ investigated a mouse model of later-life deafness that is caused by a mutant version of the Tmcl gene. This mutation is identical to one in the human version of the gene that is linked to deafness. Hearing loss is accompanied by the death of inner-ear hair cells that sense sound using their ciliary projections. The authors injected the ears of newborn mice with gene-editing components: the nuclease enzyme Cas9 that can cut DNA, and a guide RNA that targets Cas9 to the mutant version of Tmc1 in hair-cell nuclei. These were packaged in a lipid droplet that fuses with cells to enable the gene-editing components to enter. The mutant version of $\mathrm{Tmc} 1$ has an adenine nucleotide (A, highlighted in red in the mutant nucleotide sequence) at a position that is a thymidine nucleotide $(\mathrm{T})$ in the wild-type version. Gene editing selectively inactivated the mutant version of the gene through mechanisms such as nucleotide deletion. Edited cells express only the wild-type Tmcl protein (white) and don't express the mutant version (red). 
of RNA that binds to both the enzyme and the target $\mathrm{DNA}^{9}$. This approach is also known as CRISPR-Cas gene editing. The guide RNA matches the mutant but not the wild-type gene, enabling Gao and colleagues to solve the problem of ensuring that the mutant form of the gene is cut whereas the wild-type version is left untouched.

Another challenge was to get Cas9 into the inner ear. In vivo gene-editing approaches often rely on viruses to introduce nucleaseencoding sequences into the organism being edited $^{10,11}$. However, Gao and colleagues reasoned that, when the nuclease has done its job in the cell, it will no longer be required, so introducing the protein itself should suffice. They turned to a technique they had used previously ${ }^{12}$, in which they packaged Cas 9 protein bound to its guide RNA in a type of lipid droplet that can fuse with cells, enabling the editing machinery to enter. The authors injected these droplets into the inner ear of newborn Beethoven mice.

The inner ears of unedited adult Beethoven mice were barren of hair cells; however, their gene-edited adult siblings had inner-ear hair cells that were almost indistinguishable in shape and number from those in wild-type mice. The edited animals could be startled by a sudden loud noise, whereas their unedited siblings could not. More-sophisticated measurements also confirmed that hearing improved as a result of gene editing. Encouragingly, the engineered nuclease seems to have stayed true to its design and did not create undesired genetic changes of concern in the DNA of the hair cells.

A modest fraction of cells were edited. The authors propose that this low proportion of edited cells resulted in a beneficial 'halo'-like effect on neighbouring unedited cells that still contained the mutant form of the gene, preventing the death and degeneration of these neighbouring cells. Although the mechanism underlying this proposed halo effect is unclear, the finding offers encouragement for the clinical adoption of this approach, because it suggests that the genetic repair of all hair cells is perhaps not needed to achieve a beneficial effect on hearing.

Gao and colleagues' work provides an essential first step towards moving this type of approach nearer to the clinic by providing evidence that it is safe and effective in an animal that has a similar genetic mutation and comparable hearing loss to those in humans. How long could it be before individuals with this TMC1 mutation might be treated using gene editing? One reason for optimism comes from the pace at which other gene-editing approaches have reached the clinic.

To give just a few examples from clinical trials, the gene CCR5 has been inactivated in immune-system cells using a type of enzyme called a zinc-finger nuclease to try to reduce the viral load in people infected with
$\mathrm{HIV}^{13}$. Immune cells have also been edited to generate cancer-targeting cells ${ }^{14}$. However, these techniques required cells to be removed from the patient's body for gene editing and then replaced. Ear cells cannot be removed, so a direct in vivo approach is needed, which is even more challenging to achieve than ex vivo gene editing.

Encouragingly, such in vivo gene editing (for a different condition) has been performed in a clinical trial using zinc-finger nucleases ${ }^{15}$, and the work leading up to that ${ }^{16}$ makes clear the next steps for Gao and colleagues' approach. A nuclease must be found that has clinical-grade potency and specificity in human cells. Lipids must be identified that can be safely injected along with the nuclease into the human inner ear. Next, this nuclease must be tested for safety in larger animals, such as primates. An in vivo virus-based gene therapy for direct injection into the eye ${ }^{17}$ has been recommended for approval in the United States, and that work provides a road map for the scientific, medical and commercial considerations that need to be taken into account when moving to the clinic.

In 1902, the physician Archibald Garrod initiated the first study that demonstrated a link between a gene and a disease. Since then, more than 5,000 diseases have been linked to single-gene changes. However, without the tools to modify disease-causing forms of genes, geneticists have often been unable to see their knowledge put to use for clinical benefit. The progress being made with genome editing is changing this. Although Beethoven never heard his famous Ode to Joy, it could be that thanks in no small part to his murine namesake's fateful encounter with Cas9 - we are getting closer to the day when individuals with deafness-causing mutations can be treated by gene editing to prevent hearing loss.

Fyodor Urnov is at the Altius Institute for Biomedical Sciences, Seattle, Washington 98121, USA.

e-mail:urnov@altius.org

1. Gao, X. et al. Nature 553, 217-221 (2018).

2. Fettiplace, R. \& Kim, K. X. Physiol. Rev. 94, 951-986 (2014).

3. Kawashima, Y. et al. J. Clin. Invest. 121, 4796-4809 (2011).

4. Kurima, K. et al. Nature Genet. 30, 277-284 (2002).

5. Zhao, Y. et al. PLoS ONE 9, e97064 (2014).

6. Vreugde, S. et al. Nature Genet. 30, 257-258 (2002).

7. Pan, B. et al. Neuron 79, 504-515 (2013).

8. Carroll, D. Annu. Rev. Biochem. 83, 409-439 (2014)

9. Jiang, F. \& Doudna, J. A. Annu. Rev. Biophys. 46, 505-529 (2017).

10.Li, H. et al. Nature 475, 217-221 (2011).

11.Ran, F. A. et al. Nature 520, 186-191 (2015).

12.Zuris, J. A. et al. Nature Biotechnol. 33, 73-80 (2014).

13.Tebas, P. et al. N. Engl. J. Med. 370, 901-910 (2014).

14.Qasim, W. et al. Sci. Transl. Med. 9, eaaj2013 (2017) 15.https://clinicaltrials.gov/ct2/show/NCT03041324

16.Sharma, R. et al. Blood 126, 1777-1784 (2015).

17. Russell, S. et al. Lancet 390, 849-860 (2017).

This article was published online on 20 December 2017.

\section{MATERIALS SCIENCE}

\section{Rule-breaking perovskites}

A material from the perovskite family of semiconductors emits light much more efficiently than expected. The explanation for this anomalous behaviour could lead to improvements in light-emitting technology. SEE LETTER P.189

\section{MICHELE SABA}

$\mathrm{W}$ hen a semiconductor absorbs light, a particle-like entity called an exciton can be produced. Excitons comprise an electron and a hole (the absence of an electron), and have two possible states: singlet and triplet. Triplet states were thought to be poor emitters of light, but, on page 189, Becker et al. ${ }^{1}$ report that semiconductors known as lead halide perovskites have bright triplet excitons. The results could signify a breakthrough in optoelectronics because triplet states are three times more abundant than singlet states ${ }^{2}$ and currently limit the efficiency of organic light-emitting diodes ${ }^{3}$.
Conventional wisdom holds that triplet states are dark because of the spin selection rule $^{4}$, which forbids electrons from changing their intrinsic angular momentum (spin) during an optical transition - the process in which an atom or molecule switches from one energy state to another by emitting or absorbing light. The rule is taught in quantum-mechanics classes when atomic transitions are first introduced, and is so general that one might think that it is written in stone. Fortunately, there are loopholes that can be exploited.

The search for emissive triplet states has focused on a certain principle of quantum mechanics: if an electron's spin is coupled 